\title{
Research Paper \\ Prevalence of Elder Abuse in Iran: A Systematic Review and Meta Analysis
}

\author{
*Maziyar Molaei ${ }^{1}$, Korosh Etemad ${ }^{2}$, Parisa Taheri Tanjani ${ }^{3}$ \\ 1. Department of Epidemiology, School of Health, Shahid Beheshti University of Medical Sciences, Tehran, Iran. \\ 2. Environmental and Occupational Hazards Control Research Center, Shahid Beheshti University of Medical Sciences, Tehran, Iran \\ 3. Department of Internal Medicine, School of Medicine, Shahid Beheshti University of Medical Sciences, Tehran, Iran.
}

Received: 07 Jan. 2017

Accepted: 18 Apr. 2017

Keywords:

Elderly Maltreat-

ment, Prevalence,

Meta-analysis, Iran

\section{A B STRACT}

Objectives Abuse of older people is a major issue in societies that can reduce self-confidence, generate feelings of hopelessness and apathy, and cause psychological disability in the elderly. The purpose of this study is to determine the prevalence of elder abuse in Iran.

Methods \& Materials This study is a systematic review and meta-analysis, in which all articles about elderly abuse in Iran since 2005-2014 were searched using online databases such as MEDLINE, Google Scholar, SID, Web of Science, and Magiran. Their data were collected and analyzed using the Comprehensive Meta-Analysis (V 2.0, Biostat) software.

Results The highest prevalence (87.8\%) of elder abuse was found in Tehran (Manochehry et al, 2007), and the lowest prevalence (17.1\%) was found in Shahrekord (Hoseinei et al, 2014). The overall elder abuse prevalence in Iran was 56.4\% with confidence interval 95\% (35.1-75.5\%).

Conclusion The overall elder abuse prevalence in Iran is much more than that reported by foreign studies. This difference could be related to small sample size and culture variety in the various communities. The prevalence of elder abuse in Iran is much more than that in the world.

\section{Extended Abstract}

\section{Objectives}

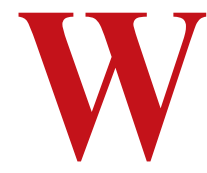

ith the advancement of medical science, improvement in nutrition quality, and prevention of many diseases over the past few decades, the length of human life has increased. Currently, a significant portion of the population of many countries constitutes the elderly. But, according to some sociologists and health professionals, societies are increasingly facing elderly maltreatment, which includes physical, sexual, psychological and emotional abuse; negligence; abandonment; and financial exploitation [1]. In Iran, according to the letter of approval of the Cabinet of Ministers No.
3478C527330 on 04.14.2004 concerning taking necessary measures to prevent the use of violence against the elderly in the family and society, no steps have been yet taken to determine the extent of this problem [2]. Therefore, the purpose of this study is integrating different studies conducted in Iran and achieving a common outcome from the breadth of the problem, i.e., the general prevalence of elderly maltreatment.

\section{Methods \& Materials}

This study was conducted using systematic review and meta-analysis in which 55 articles concerning elderly maltreatment in Iran from 2005 to 2015 in SID, Google scholar, Magiran, Iranmedex, Pubmed were gathered. Words such as elderly maltreatment or "mistreatment" 
or "neglect" and the "prevalence" were considered as keywords for searching articles. Inclusion criteria for the study were articles that discuss the prevalence of the elderly maltreatment, studies on the prevalence of elderly maltreatment in people aged 60-65 or older than 65, studies in Persian or English, studies used cross-sectional method, articles that had the same definition of the elderly maltreatment, articles conducted from 2005 to 2015, as well as articles that earn at least $20 \%$ of the score of the STROBE checklist. For each study, the following information was extracted separately: name of the first author, year of study, year of publication, place of research, type of target population, type of study, total target population, and the overall prevalence of each study.

Ten articles related to the topic were selected. In the next step, these articles were evaluated by the STROBE checklist and then entered in the meta-analysis phase. Some articles did not consider a general prevalence and some did not consider all aspects of the prevalence; hence, fewer articles were entered into the meta-analysis. Analysis was done using the Comprehensive Meta-Analysis (V. 2, Biostat) software. The value of the index in each study and the estimated overall estimate (prevalence) were obtained with random model or fixed model with $95 \%$ confidence interval and a significant level of $0.05 \%$, and they are displayed using the FORST PLOT accumulation chart. Heterogeneity between studies was examined using I 2 test, and since the number of articles is less than 10, propagation does not apply and its charts are not drawn. This article is approved by the Ethics Committee of Shahid Beheshti University of Medical Sciences of Tehran.

\section{Results}

Total number of participants among all the articles entered into the meta-analysis was 3436 . All studies were conducted on both genders. Information on the prevalence of elderly maltreatment in each article were obtained through face-to-face interviews and completed questionnaires. Since the data collection method was the same for all articles, all articles have the same definition of elderly maltreatment and had various aspects. Sampling was also carried out randomly, indicating that these differences in prevalence rates seem to be real. As seen in Table 1, the most common temporal prevalence was maltreatment with the elderly $(87.8 \%)$ in the study by Manouchehri et al. [3], which was conducted in 2008 on a population of over 60 years old people in Tehran parks, and the least one was related to the study by Hosseini et al. (17.1\%) in 2015 in Shahrekord [9].

With a simple review, it seems that among the various aspects of elderly maltreatment, emotional abuse is the most frequent type of maltreatment and its value ranges from $9.6 \%$ [23] to $84 \%$ [7]. Other aspects of the elderly maltreatment also vary in various studies with highly extensive prevalence rates. The prevalence of physical abuse ranges from $2.8 \%$ [4] to $35.9 \%$ [9], financial malpractice is $5.8 \%$ [23] to $40 \%$ [7], and psychological misconduct is $12.4 \%$ [10] to $59.8 \%$ [11]. The prevalence rate of neglecting is between $4.5 \%$ [9] and $68 \%$ [7] and rejection rate is between $3.7 \%$ [20] up to $23 \%$ [9].

To combine an indicator of the prevalence of elderly maltreatment in the studies investigated, a fixed model was used due to the heterogeneity of studies (inhomogeneity index I2 $=98.97$ for the overall prevalence of elderly maltreatment, physical, financial, emotional, psychological abuse, rejection and negligence were 91.98, 98, 99, 96, 96 and 98, respectively). For this reason, the random model was used to combine the indices.

Using the Random Effect model, the overall rate of prevalence in Iran in this study was $56.4 \%$ with a con-

Table 1. Results of the frequency elderly maltreatment prevalence and its subcategories

\begin{tabular}{ccccc}
\hline Row & Index & Number of Articles & Frequency of Prevalence (\%) & Confidence Interval of 95\% (\%) \\
\hline 1 & $\begin{array}{c}\text { General prevalence of } \\
\text { elderly maltreatment }\end{array}$ & 8 & 56.4 & $35.1-75.5$ \\
\hline 2 & Physical & 9 & 13.1 & $7.8-21.2$ \\
\hline 3 & Financial & 8 & 19.7 & $12.9-28.9$ \\
\hline 4 & Emotional & 7 & 30.7 & $51.1-15.8$ \\
\hline 5 & Psychological & 7 & 25.4 & $14.5-40.6$ \\
\hline 6 & Exclusion & 7 & 11.7 & $8-16.8$ \\
\hline 7 & Negligence & 8 & 25.1 & $13.6-41.7$ \\
\hline
\end{tabular}


fidence interval of $95 \%$ (35.1-75.5). In this model, the highest prevalence of the type of elderly maltreatment in Iran was related to emotional abuse $(30.7 \%)$, followed by mental ill-treatment (25.45\%). Temporal prevalences of other aspects of elderly maltreatment including physical and financial misconduct, negligence, and exclusion were $13.1 \%, 19.7 \%, 25.1 \%$, and $11.7 \%$, respectively (Table 1 ).

\section{Conclusion}

The prevalence of maltreatment towards the elderly in Iran is more than that in the world, and perhaps the high prevalence of the maltreatment cannot be attributed only to the deterioration of this phenomenon. This is because it is one of the social problems which are undoubtedly influenced by various factors. Since the problem of maltreatment of the elderly addressed in the global community, especially in our society, is a new and unknown phenomenon and even we are sometimes faced with resistance in this area in form of denying or disregarding this phenomenon, therefore, conducting this study, expressing the extent of the problem and confirming the high prevalence of maltreatment in Iran, a turning point can be reached for further investigation and research concerning the elderly maltreatment and the various aspects of it including epidemiology, related factors, risk factors, prevention methods and intervention and so on. This research can act as a foundation for the next research. It is noteworthy that there are certainly more cases of maltreatment which have not been mentioned for various reasons and the items mentioned are just the tip of the iceberg as many cases remained to be investigated.

\section{Acknowledgments}

This paper is extracted from the first author in the Department of Epidemiology, School of Health, Shahid Beheshti University of Medical Sciences, Tehran.

\section{Conflict of Interest}

All authors certify that this manuscript has neither been published in whole nor in part nor being considered for publication elsewhere. The authors have no conflicts of interest to declare. 


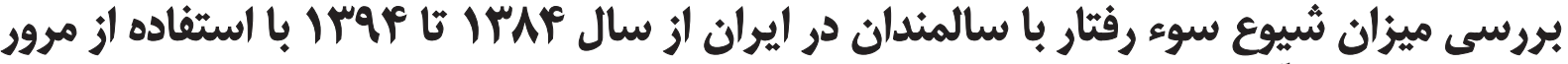 ساختاريافته ومثآناليز
}

\author{
"مازيار مولايى'، كورش اعتماد'، بريسا طاهرى تنجانى"

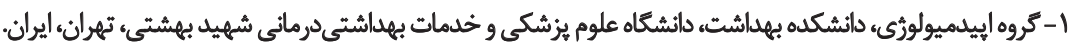

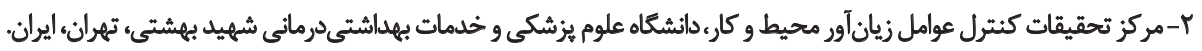

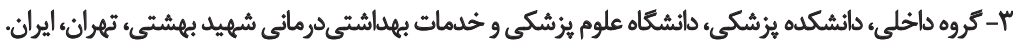

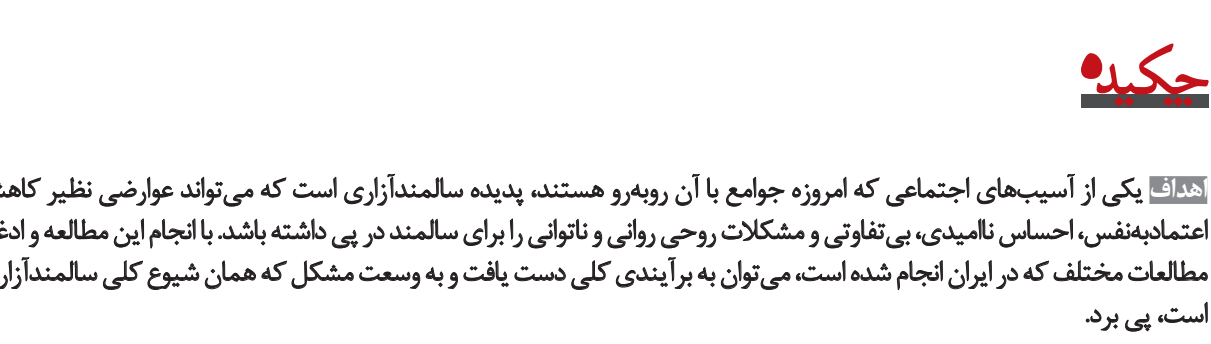

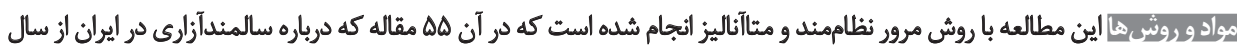

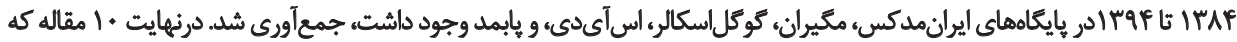

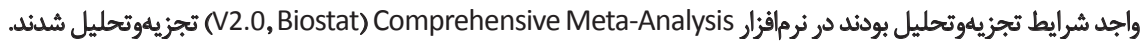

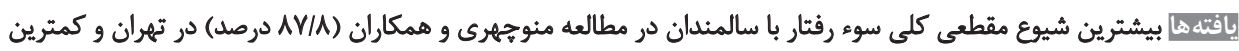

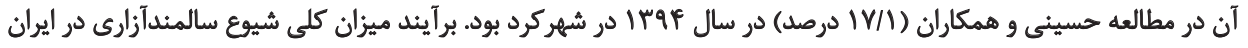

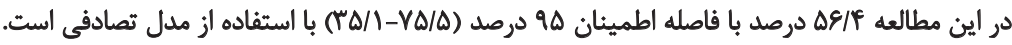

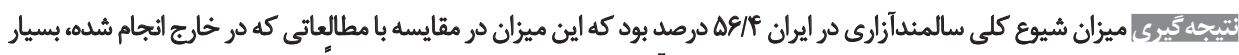

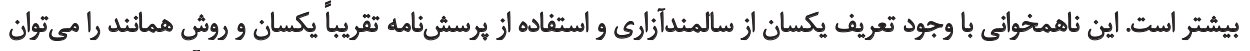

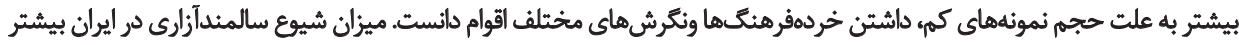

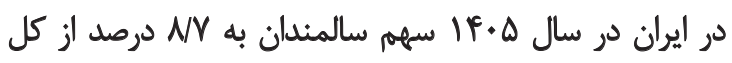

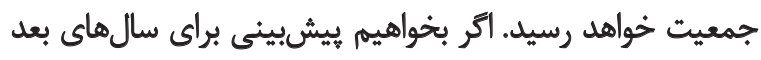

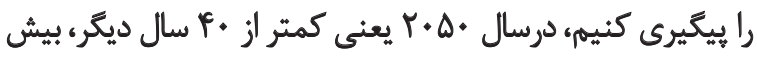

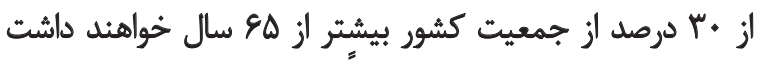
و هرم سنى در سال •ه.r، كاملاً سال خورده و استوانهائشكل

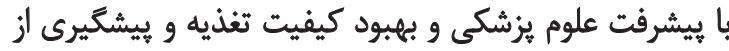

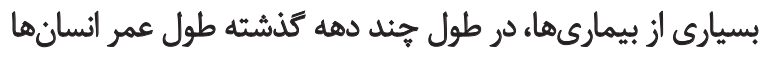

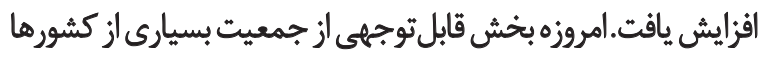

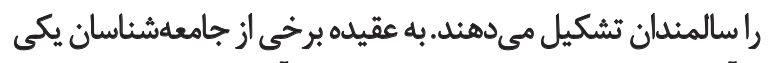
از آسيبهاى اجتماعى كه امروزه جوامع با آن روبهرو هستيند، بـانديده سالمندآزارى است. أست.

طبق تعريف مركز كنترل و بيشعيرى از بيمارى بها'، سوءرفتار باريا

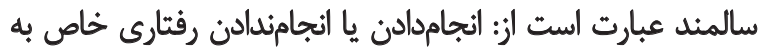

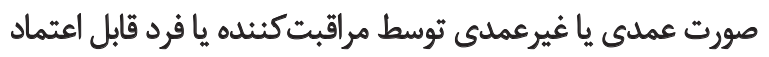

1. Center of Disease Control (CDC) 
بودند؛ مطالعاتى كه به زبان فارسى يا انتليسى بودند؛ مطالعاتى كه

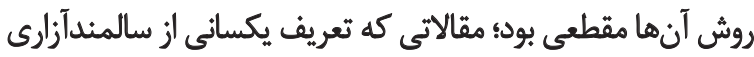

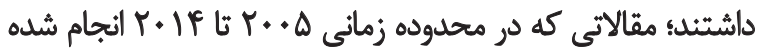

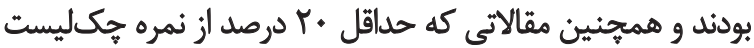

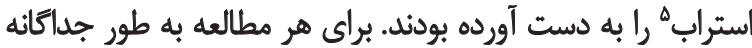

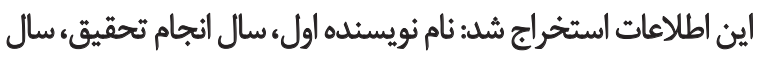

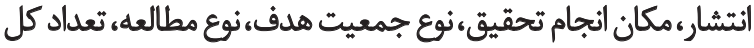
جمعيت هدف و شيوع كلى هر مطالعه.

در همه مقالاتي كه در جدول شماره ا ذأكر شده است، اطلاعات

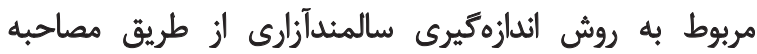

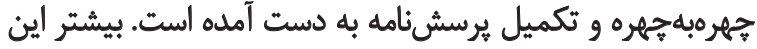

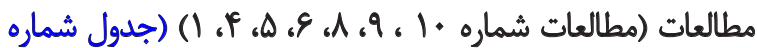

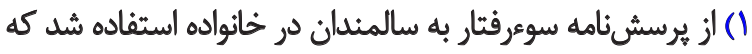

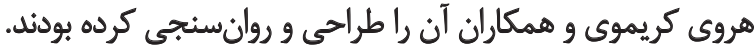

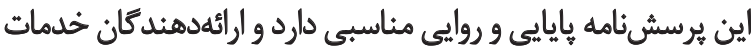

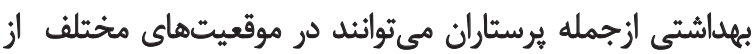

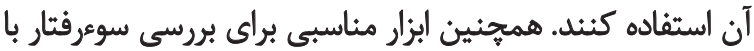
سالمند در خانوادههاى ايراني است.

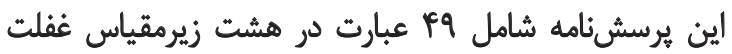

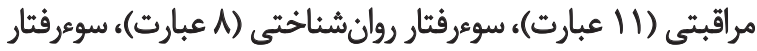

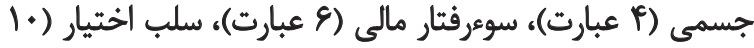

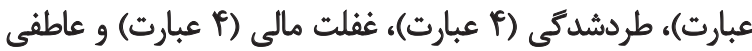
(r)

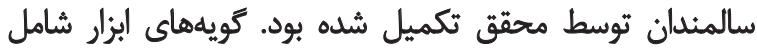

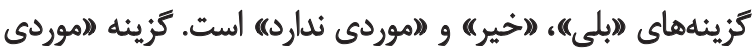

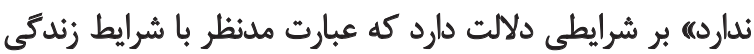

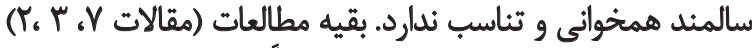

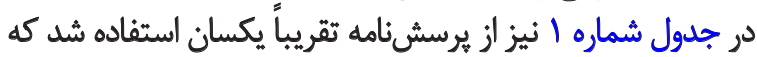

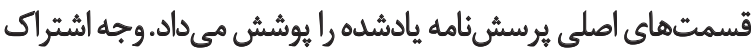

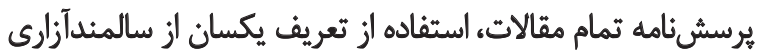

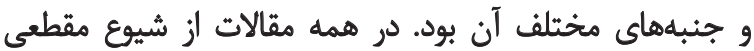

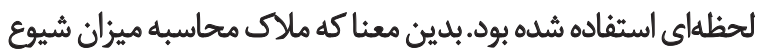

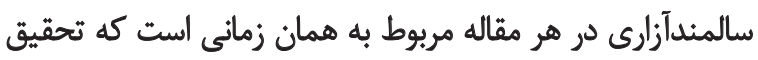

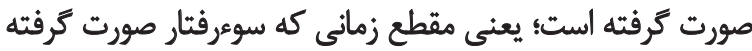

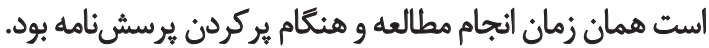
از خكليست استراب مخصوص مطالعات مقطعى براى سنجش

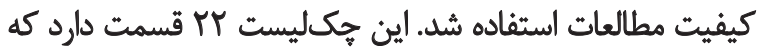

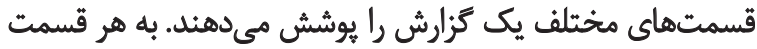

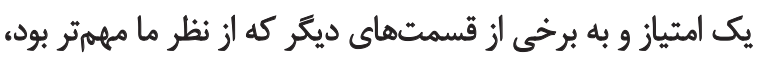

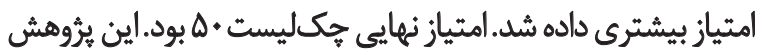

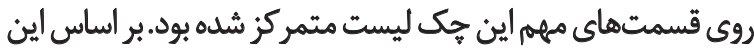

5. Strobe

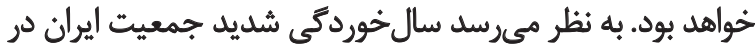

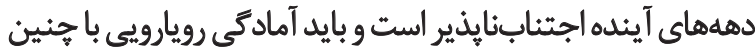

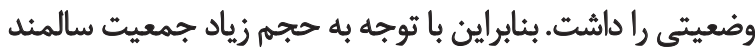

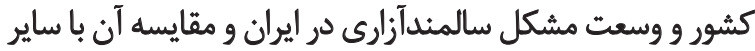

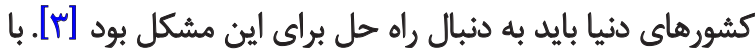

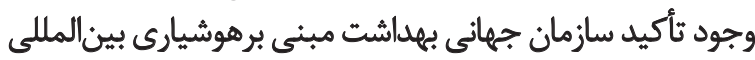

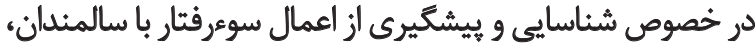

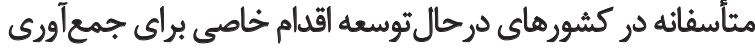

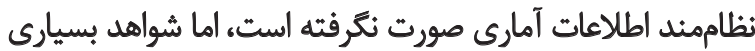

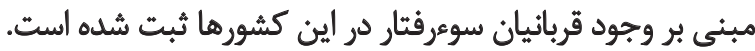

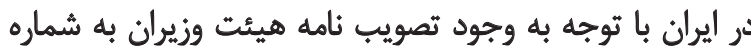
دات

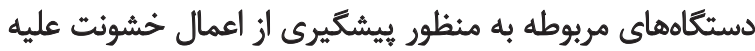

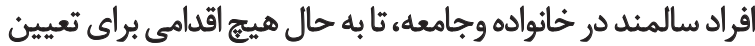

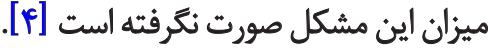

تحقيقات كشورى انجامشده در خصوص سالمندان در سال

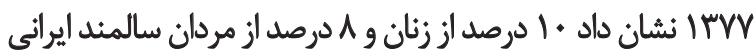

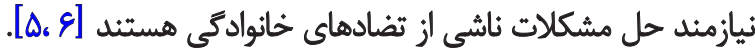

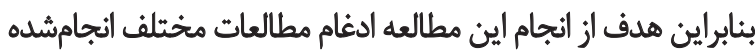

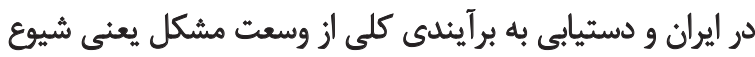
كلى سالمندآزارى است.

\section{روش مطالعه}

اين مطالعه با روش مثآآناليز انجام شد كه در آن تمامى مقالاتى ساري

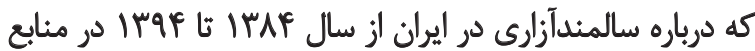

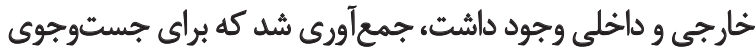

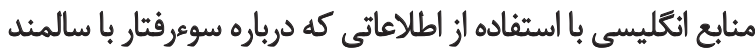

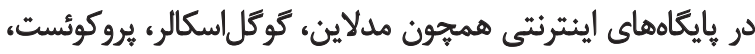

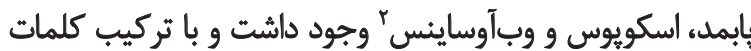

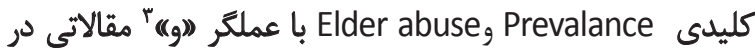

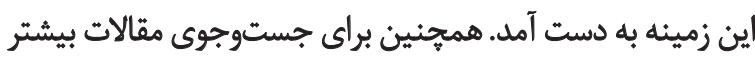

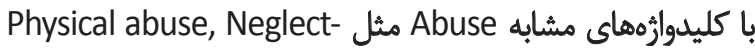
financial, Material abuse, Psychological, Verbal abuse, Violation elder abuse, Mistreatment, and Violance

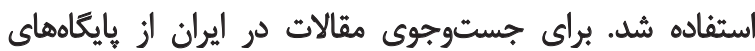

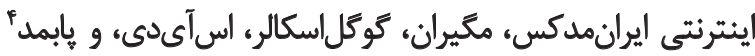

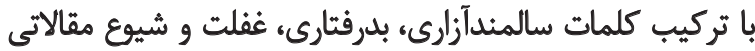

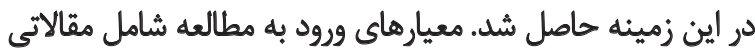

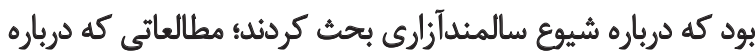

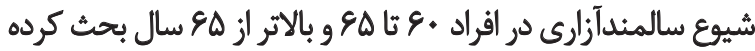

2. Medline, Google Scholar, Proquest, Pubmed, Scopus, Web of Science

3. And

4. SID, Google scholar, Magiran, Iranmedex, Pubmed 
در جستحوى اوليه همثاله داخلى در بإيكاههائ

SID-MAGIRAN-GOOGLE SCHOLARE

10 مقاله بى ارتّباط PUBMED-

تشخيص داده شيلند.

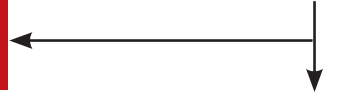

با برسى عناوين مقالات + P هقاله يافت شيد كه بر أعاس

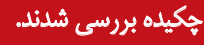

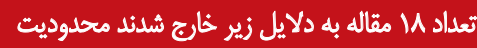

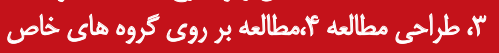

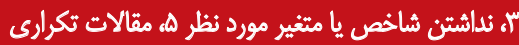

ditar

با بررسى جكيده مثالات با مقاله با متن كامل بررنسى كرديد.

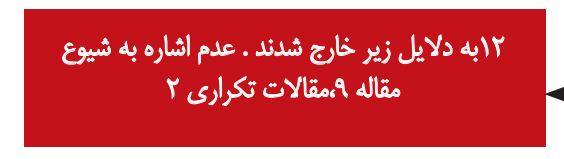

\section{تعلد • أمقاله أز نظر كيفي بروسي كرديلند}

تعداد ما هقاله قابليت ورود به هرحله آثاليز را دارئد

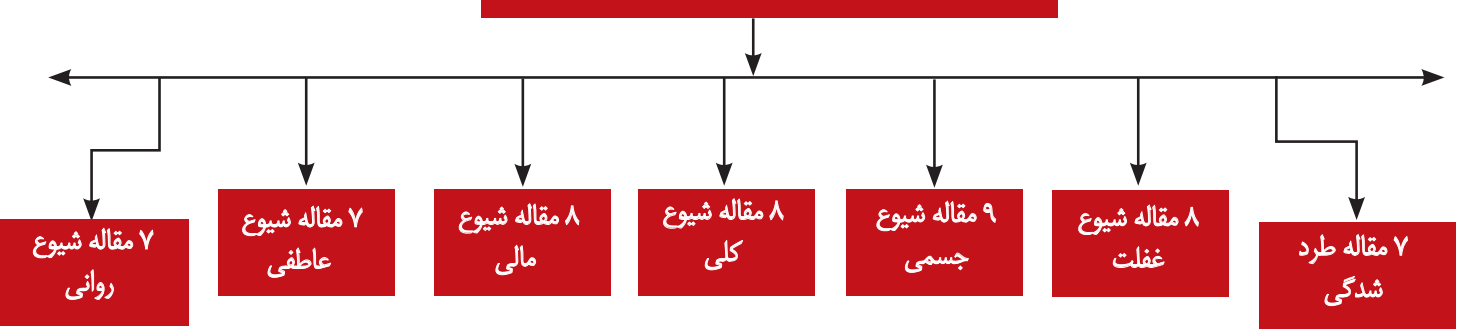

L

تجزيهوتحليل بانرمافزار جامع فراتحليل \$انجام شد.مقدار شاخص

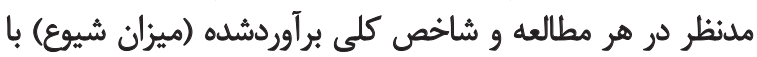

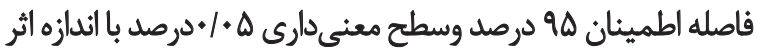

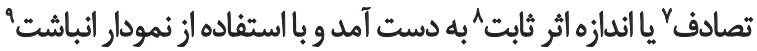

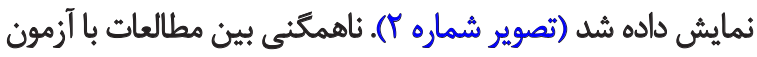

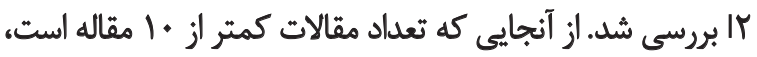
تورش انتشار كاربردى ندارد و نمودار آن رسم نمى نعالات كمترد.

يافتهها

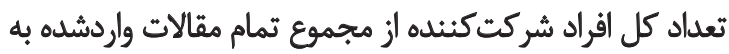

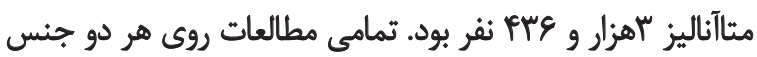

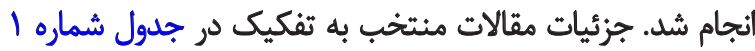

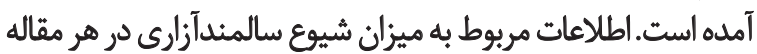

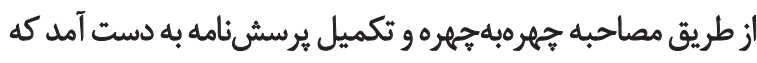

6. Comprehensive Meta-Analysis (V 2.0, Biostat) software

7. Model random

8. Fix model

9. Forest Plot
تصوير ا. مراحل ورود به متا آناليز ميزان شيوع سالمندآزارى در ايران

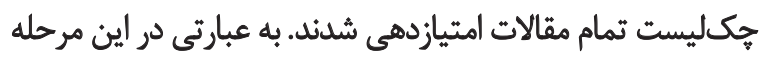

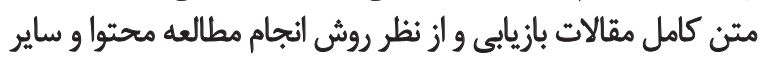

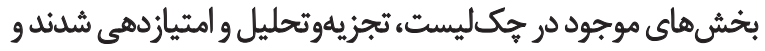
مطالعات بر اساس كيفيت طبقهبيندى شدند.

حداقل امتياز قابل قبول براى ورود مقالات · ب درصد از امتياز

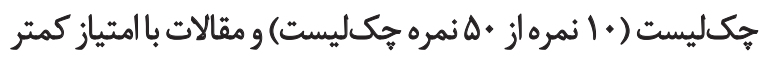

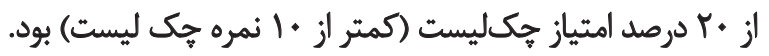

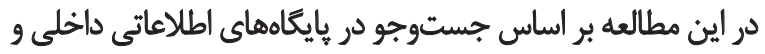

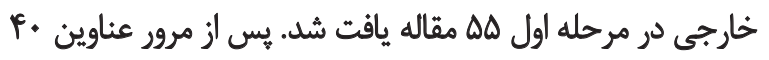

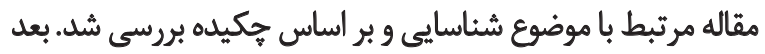

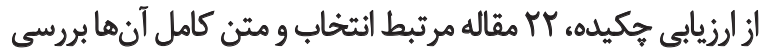

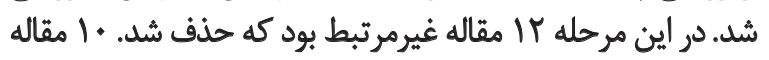

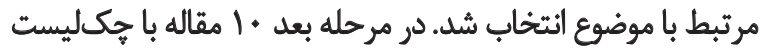

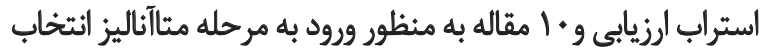

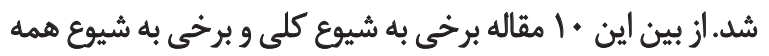

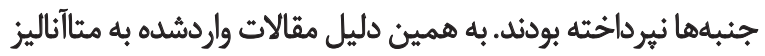
كمتر از · إ مقاله هستند (تصوير شماره ا). 


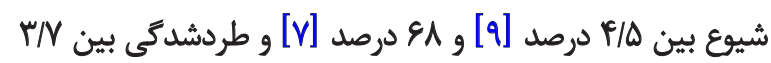

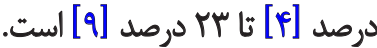

براى تركيب شاخص ميزان شيوع سالمندآزارى در مطالعات

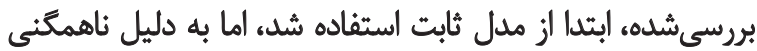

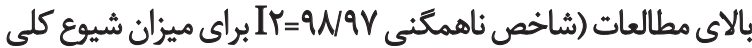

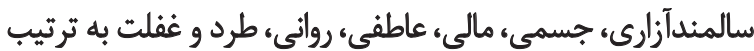
91 مدل مادفى استفاده شد.

برآيند ميزان كلى شيوع سالمندآزارى در ايران در اين مطالعه

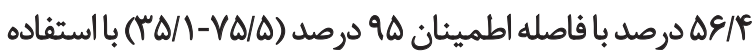

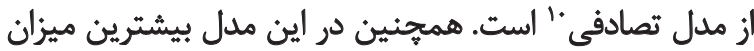

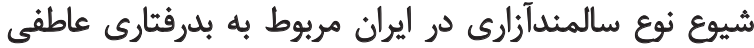

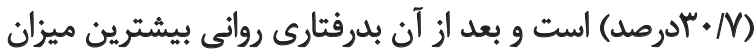

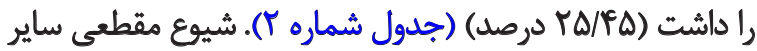

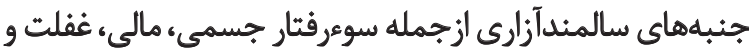

10. Random effect

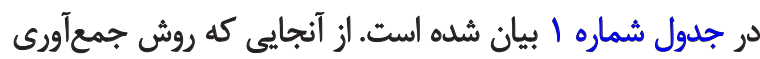

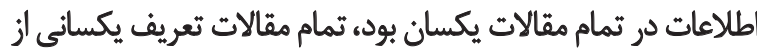

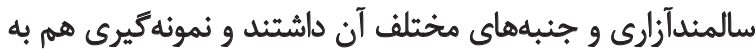

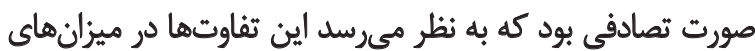

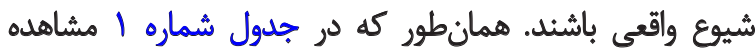

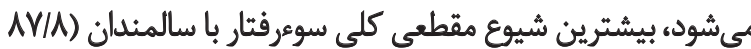

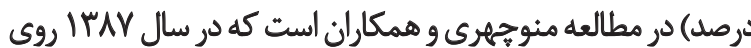

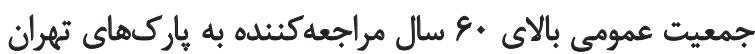

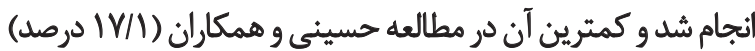

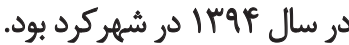

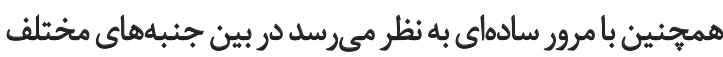

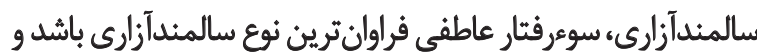

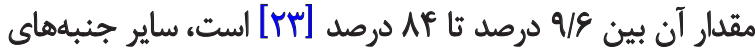

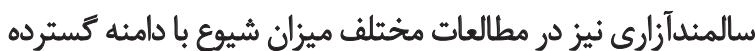

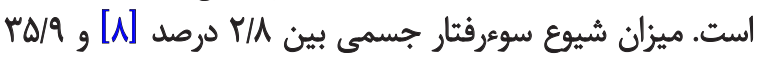

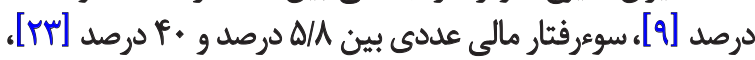

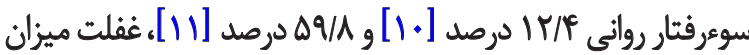

جدول ا. اطلاعات مطالعات منتخب در زميئه ى شيوع سالميدآزارى در ايران

\begin{tabular}{|c|c|c|c|c|c|c|c|c|c|}
\hline مقالمند زهانى سكة & دوصد & شيوع & تمونه & مطالعه & جمعيت هدف & مكان & زمان & نام نويسنده & رديف \\
\hline (هر زمان أجام مطالعكرى) & 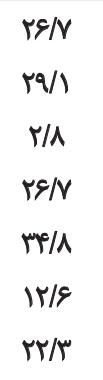 & 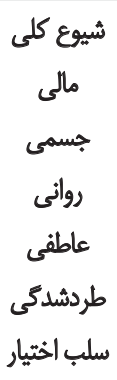 & rev & مقطعى & جمعيت بالاي .9 & ايران/كلاله & 1491 & 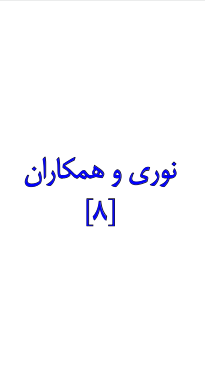 & 1 \\
\hline در زمان انجام مطالعه & $\begin{array}{l}I V / N F \\
r / \Delta \\
I F / T \\
r \Delta / q \\
I \Delta / r \\
r\end{array}$ & شفيو كلى & $\varphi .$. & مقطعى & جمعيت بالاى 9. جمال & وبختارمحال & Irap & $\begin{array}{c}\text { حسينى و همكاران } \\
\text { [ همان }\end{array}$ & $r$ \\
\hline 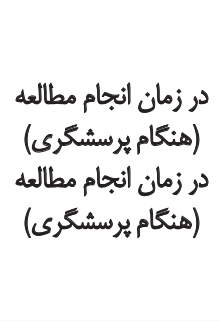 & $\begin{array}{l}\text { ذكرنشده } \\
r / N \\
r \Delta \\
10 \\
18 / 9 \\
\text { Ir }\end{array}$ & 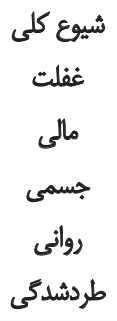 & YAS & مقطعى & جمعيت عمومى بالاى & ايران/|هواز & ITE & كريمى و الهمى [r]] & $r$ \\
\hline 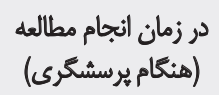 & va/s & شيوع كلى & $r \Delta$. & مقطعى & جمعيت عمومى بالاى .8 بال & ايران /يزد & שפוזו & 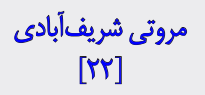 & $r$ \\
\hline
\end{tabular}




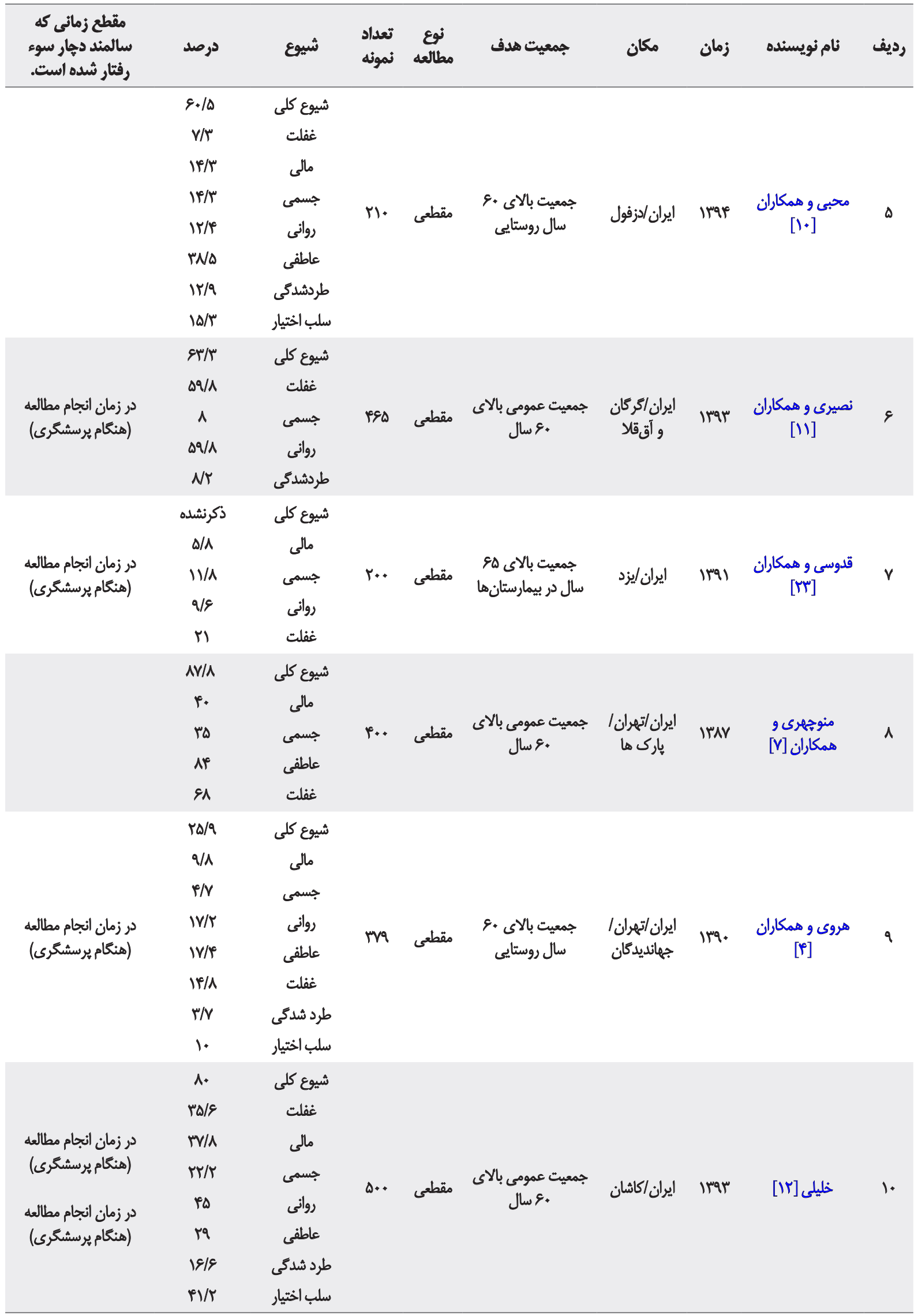


جدول ז. دادههاي كلى تحليل مطالعات منتخب در مئا آناليز شيوع سالمندآزارى در ايران

\begin{tabular}{|c|c|c|c|c|c|c|c|c|c|c|c|c|c|c|}
\hline \multicolumn{3}{|c|}{ TauY } & \multicolumn{3}{|c|}{ عدم يكنواختى } & \multicolumn{4}{|c|}{ آزمون فرضيه } & \multicolumn{4}{|c|}{ اندازه اثٔر با فاصله اطمينان 90} & \multirow{2}{*}{ مدل } \\
\hline Tau & واريانس & استاندارد أن & $\begin{array}{c}\text { Tau } \\
r\end{array}$ & IT & $P$ & $\mathrm{df}(\mathrm{Q})$ & $\mathrm{Q}$ & $P$ & Z & بالا & حايين & كلى & مقالات & \\
\hline ITOI & - $N A 1$ & - IMP & $1 / \Delta \neq \Delta$ & WaV. & $\%$ & 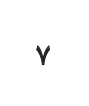 & EN/MI & .100 & $\begin{array}{l}\text { D/MP } \\
\text { - /AVA }\end{array}$ &.$/ 0 V 9$ & . & $\begin{array}{l}.10 \Delta A \\
.10 A P\end{array}$ & $\begin{array}{l}\wedge \\
\wedge\end{array}$ & تصليت \\
\hline
\end{tabular}

记

جدول با. نتايج حاصل ازٔ ميزان شيوع سالمثدآزارى و زيركروههاى آن در ايران

\begin{tabular}{|c|c|c|c|c|}
\hline قاصله اطمينان ه9 (درصد) & ميزان شيوع مقطعى (د, صد) & تعداد مقاله & شاخص & رديف \\
\hline$r \Delta / 1-V \Delta / \Delta$ & $\Delta \& / N^{e}$ & $\wedge$ & شيوع كلى سالمثدآزارى & 1 \\
\hline$V / A-Y I / T$ & $\mid m / 1$ & 9 & جسمي & r \\
\hline IY/Q-YNQ & $19 / N$ & $\wedge$ & 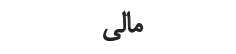 & r \\
\hline$|\Delta / A-\Delta| / \mid$ & $r \cdot N$ & 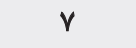 & عاطفى & $p$ \\
\hline$\| \varphi / Q-\varphi+18$ & $r \Delta / r^{\prime}$ & r & 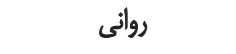 & $\Delta$ \\
\hline$A-\mid \varepsilon / 1$ & $11 / N$ & r & طردشدىى & $\varepsilon$ \\
\hline$|r / 4-r| N$ & $r \Delta / 1$ & $\wedge$ & غفلت & $\checkmark$ \\
\hline
\end{tabular}

il

Study namejyear place

\section{Statistics for each study}

Event Lower Upper rate limit limit

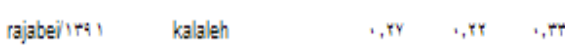

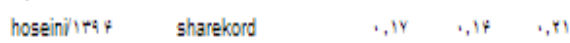

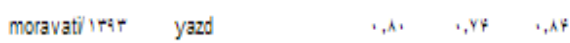

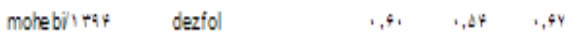

nasirylita4 gorgan\&agh ghola $\quad 1,4 \% \quad 3,49 \quad, 4,44$

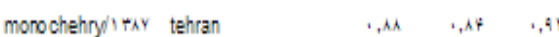

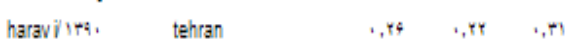

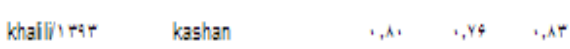

\section{Event rate and $9 \mathrm{a} \% \mathrm{Cl}$}

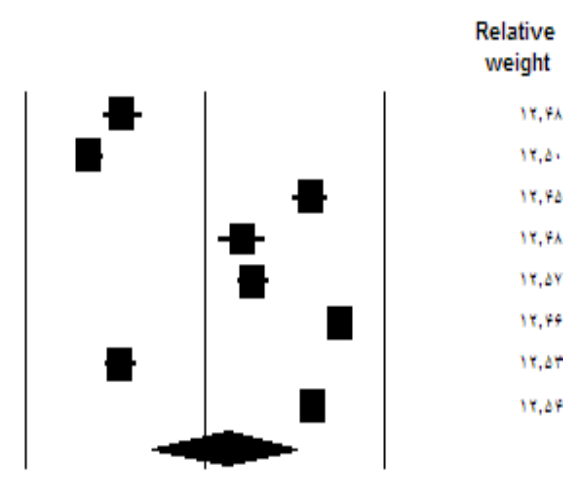

Favours A

Favours B 


\section{نتيجهَيَيرى نهايى}

ميزان شيوع سوءرفتار با سالمندان در ايران از ديكر كشورهاى

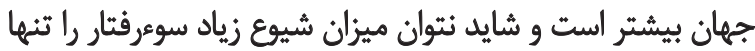

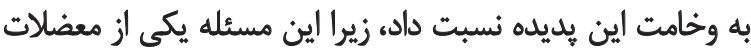

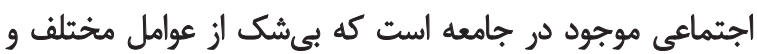

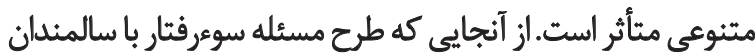

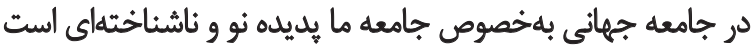

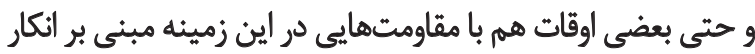

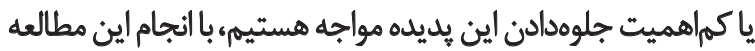

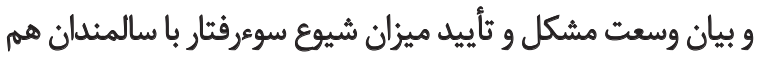

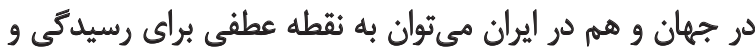

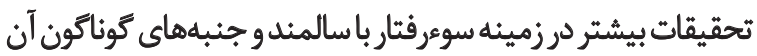

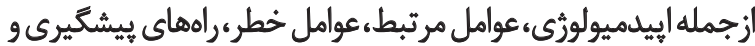

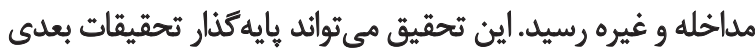

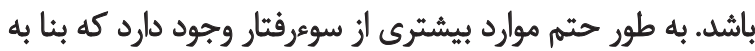

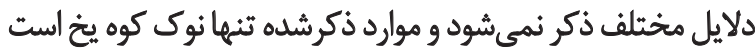
و موارد متعددى ينهان باقى مانده است.

از طرف ديكر در ايران، فرهنگ ايرانى و باورهاي سنتى همجنينان

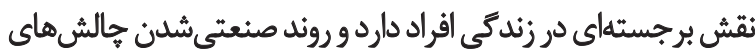

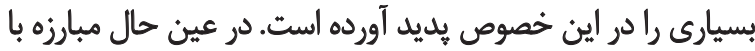

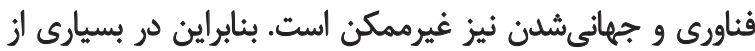

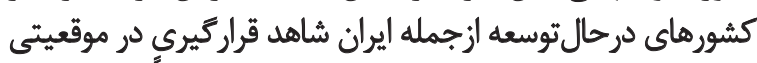

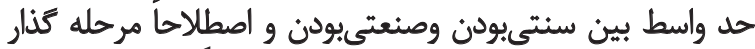

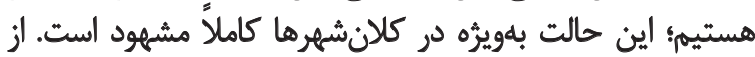

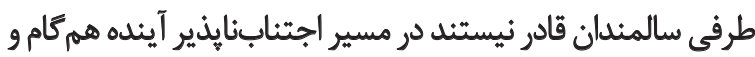

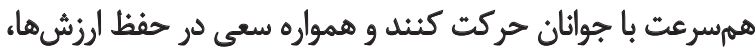

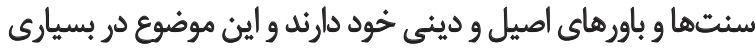

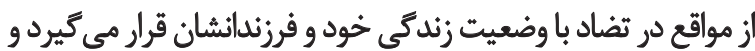
اين تضاد درواقع زمينه را براي سوءرفتار آماده مي كند.

$$
\text { تشكر و قدر داني }
$$

اين مقاله از پاياننامه كارشناسى ارشد إيدميولوزئ آقاى مازيار

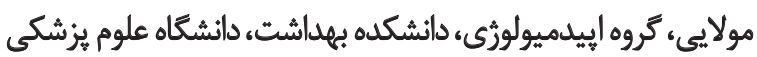

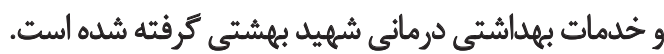

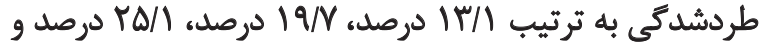

ال درصد است (جدول شماره تاب).

بحث

يافتههاى اين يثروهش نشان داد بهيترين برآورد از ميزان شيوع

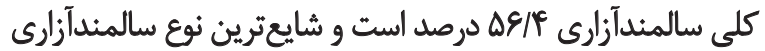

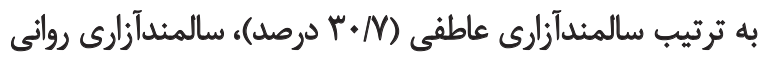

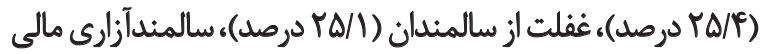

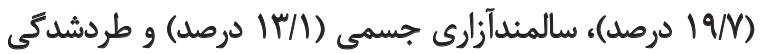

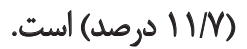

همانطور كه اشاره شد، برآورد ميزان شيوع كلى سالمندآزارى

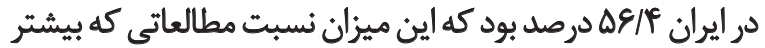

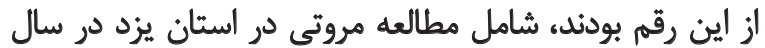

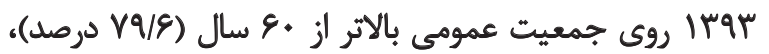

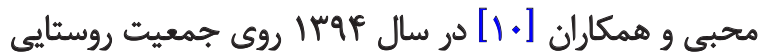

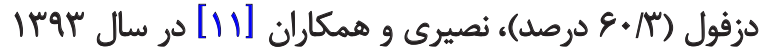

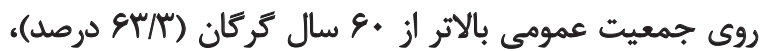

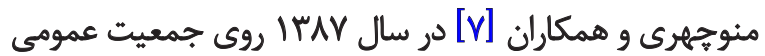

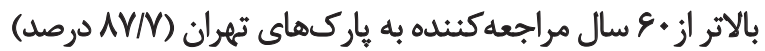

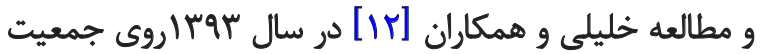

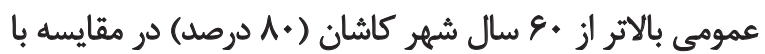

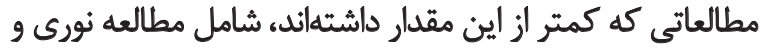

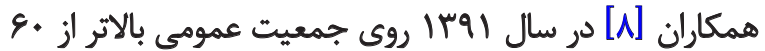

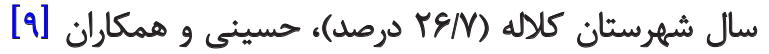

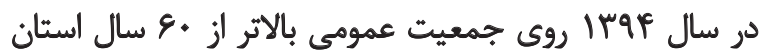

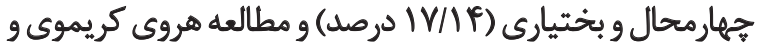

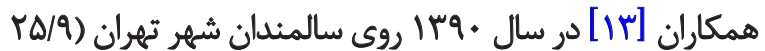

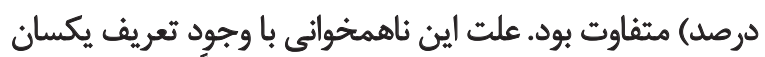

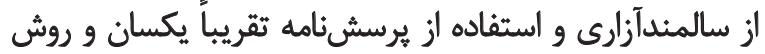

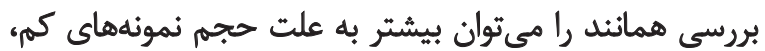

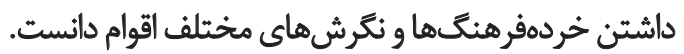

از طرف ديكر اين ميزان به مقدار قابل ملاحظهاى بيشتر از

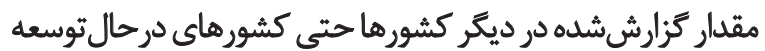

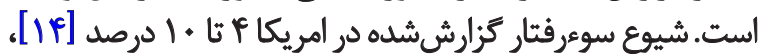

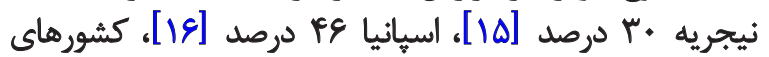

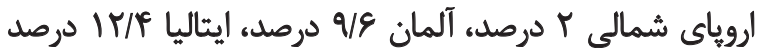

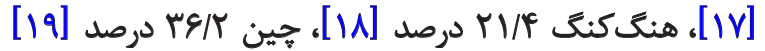

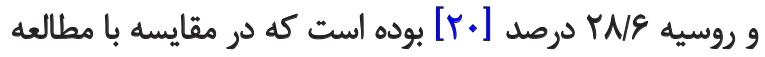

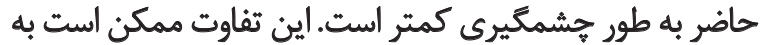

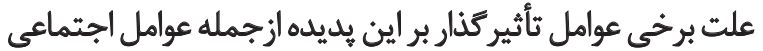

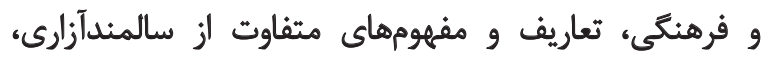

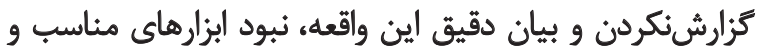

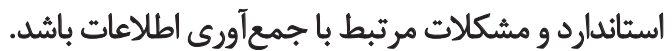




\section{References}

[1] Keyghobadi F, Moghaddam Hosseini V, Keyghobadi F, Rakhshani $\mathrm{MH}$. [Prevalence of elder abuse against women and associated factors (Persian)]. Journal of Mazandaran University of Medical Sciences. 2014; 24(117):125-32.

[2] Naughton C, Drennan J, Treacy MP, Lafferty A, Lyons I, Phelan A, et al. Abuse and neglect of older people in Ireland: Report on the national study of elder abuse and neglect, Report summary. Ireland: National Centre for the Protection of Older People. 2010.

[3] Zarghami H. [An review of ageing dynamics in iran 2011 (Persian)]. Tehran: Statistical Center of Ian; 2015.

[4] Heravi Karimooi M, Anoosheh M, Foroughan M, Hajizadeh E, Sheykhi MT. [The meaning of domestic elderly abuse concept (Persian)]. Daneshvar. 2010; 17(85):39-51.

[5] Pournaghash Tehrani S. [Assessment of domestic violence in families in Tehran (Persian)]. Daneshvar. 2006; 13:23-36.

[6] Buka P, Sookhoo D. Current legal responses to elder abuse. International Journal of Older People Nursing. 2006; 1(4):194-200. doi: 10.1111/j.1748-3743.2006.00029.x

[7] Manoochehri H, Ghorbi B, Hosseini M, Oskuyee NN, Karbakhsh $\mathrm{M}$. [Degree and types of domestic abuse in the elderly referring to parks of Tehran (Persian)]. Journal of Nursing \& Midwifery. 2009; 18(63):39-45.

[8] Nori A, Rajabi A, Esmailzadeh F. [Prevalence of elder misbehavior in northern Iran (2012) (Persian)]. Journal of Gorgan University of Medical Sciences. 2015; 16(4):93-8.

[9] Hosseini RS, Salehabadi R, Javanbakhtian R, Alijanpour Aghamaleki M, Borhani nejad VR, Pakpour V. [A comparison on elderly abuse in Persian and Turkish race in Chaharmahal Bakhtiari Province (Persian)]. Journal of Sabzevar University of Medical Sciences. 2015; 23(1):75-83

[10] Mohebi L. [Compare the amount and types of abuse towards the elderly and its impact on quality of life in Dezful city and the villages (Persian)] [MSc thesis]. Ahvaz: Ahvaz Jundishapur University of Medical sciences; 2015

[11] Nassiri H, Heravi Karimooi M, Jouybari L, Sanagoo A, Chehrehoosha M. [The prevalence of elder abuse in Gorgan and Aq-Qala cities, Iran in 2013 (Persian)]. Iranian Journal of Ageing. 2016; 10(4):162-73.

[12] Khalili Z. [The prevalence of elder abuse and its related factors in Kashan (Persian)] [MSc. thesis]. Kashan: Kashan University of Medical Sciences and Health Services; 2015.

[13] Heravi Karimoei M, Reje N, Foroughan M, Montazeri A. [Elderly abuse rates within family among members of senior social clubs in Tehran (Persian)]. Iranian Journal of Ageing. 2012; 6(4):37-50

[14] Strasser S, Smith M, Weaver S, Zheng S, Cao Y. Screening for elder mistreatment among older adults seeking legal assistance services. Western Journal of Emergency Medicine. 2013; 14(4):309 15. doi: 10.5811/westjem.2013.2.15640

[15] Cadmus EO, Owoaje ET. Prevalence and correlates of elder abuse among older women in rural and urban communities in South Western Nigeria. Health Care for Women International. 2012; 33(10):973-84. doi: 10.1080/07399332.2012.655394
[16] Pérez-Cárceles MD, Rubio L, Pereniguez JE, Pérez-Flores D, Osuna E, Luna A. Suspicion of elder abuse in South Eastern Spain: The extent and risk factors. Archives of Gerontology and Geriatrics. 2009; 49(1):132-7. doi: 10.1016/j.archger.2008.06.002

[17] Santos CM dos, Marchi RJD, Martins AB, Hugo FN, Padilha $\mathrm{DMP}$, Hilgert JB. The prevalence of elder abuse in the Porto Alegre metropolitan area. Brazilian Oral Research . 2013; 27(3):197-202 doi: 10.1590/s1806-83242013005000011

[18] Abath M de B, Leal MCC, Melo Filho DA de, Marques AP de O. Physical abuse of older people reported at the institute of Forensic Medicine in Recife, Pernambuco State, Brazil. Cadernos de Saúde Pública. 2010; 26(9):1797-806. doi: 10.1590/s0102311×2010000900013

[19] Wu L, Chen H, Hu Y, Xiang H, Yu X, Zhang T, Cao Z, Wang $Y$. Prevalence and associated factors of elder mistreatment in a rural community in People's Republic of China: A cross-sectional study. PloS ONE. 2012; 7(3):33857. doi: 10.1371/journal. pone. 0033857

[20] Heravi Karimoei M, Reje N, Foroughan M, Montazeri A. [E]derly abuse rates within family among members of senior social clubs in Tehran (Persian)]. Iranian Journal of Ageing. 2012 6(4):37-50.

[21] Karimi M, Elahi N. [Elderly abuse in Ahwaz city and its relationship with individual and social characteristics (Persian)]. Iranian Journal of Ageing. 2008; 3(7):42-6.

[22] Morowatisharifabad MA, Rezaeipandari H, Dehghani A, Zeinali A. Domestic elder abuse in Yazd, Iran: a cross-sectional study. Health Promotion Perspectives. 2016; 6(2):104-10. doi: 10.15171/ hpp.2016.18

[23] Ghodoosi A, Fallah Yakhdani E, Abedi H A. [Studying the instances of elder abuse and their relationship with age and sex in the hospitalized elderly (Persian)]. Scientific Journal of Forensic Medicine. 2014; 20 (1) :367-376 
